\title{
Radiological Exposure Device
}

National Cancer Institute

\section{Source}

National Cancer Institute. Radiological Exposure Device. NCI Thesaurus. Code C120579.

A device constructed from partially or fully unshielded radioactive material intended to expose people to significant doses of ionizing radiation without their knowledge. 